\title{
EFECTOS DE LA RELACIÓN HEMBRA: MACHO Y EDAD DE LOS REPRODUCTORES EN EL COMPORTAMIENTO REPRODUCTIVO DE LA CODORNIZ JAPONESA (Coturnix coturnix japónica)
}

\author{
EFFECTS OF MATING RATIO AND PARENTAL AGE ON REPRODUCTIVE \\ PERFORMANCE IN JAPANESE QUAIL (Coturnix coturnix japónica)
}

\author{
${ }^{1}$ Jorge Valladares Pérez y ${ }^{2}$ Marcial Estanislao Cumpa Gavidia
}

\begin{abstract}
Resumen
El objetivo del presente estudio fue evaluar los efectos de la relación de apareamiento hembra: macho y edad de los reproductores sobre la producción de huevos, fertilidad e incubabilidad de la codorniz japonesa. En este estudio, las codornices reproductoras fueron colocadas en jaulas en relaciones hembra: macho de 3:1, 4:1 y 5:1. Además, las reproductoras fueron separadas en dos grupos de 21 y 47 semanas de edad. Se utilizó el Diseño de Bloques completamente randomizado con arreglo factorial 3 x 2 (3 relaciones hembra: macho x 2 edades de reproductores). Los efectos de las relaciones de apareamiento sobre la producción de huevos, fertilidad e incubabilidad no fueron estadísticamente significativos. La edad de los reproductores no influencio sobre la producción de huevos e incubabilidad. Los resultados muestran que la fertilidad en codornices de 47 semanas de edad fue significativamente más alto comparado con las codornices de 21 semanas de edad.
\end{abstract}

Palabras clave: Relación hembra: macho, edad de reproductores, comportamiento reproductivo, codornices.

\begin{abstract}
The objective of the present study was to evaluate the effects of female: male ratio and parental age on egg production, fertility and hatchability on Japanese quails. In this study, quail breeders were placed in cages at female to male ratios of 3:1, 4:1 and 5:1. Besides, quails were separated in two groups of 21 and 47-week- old. It was used Randomized Block design with factorial arrangement 3 × 2 (3 ratios female: male x 2 parental age). The effects of mating ratio on egg production, fertility and hatchability were not statistically significant. Parental age had no influence on egg production and hatchability. Results showed that fertility in quails of 47 weeks of age was significatively higher compared to group of 21 weeks of age.
\end{abstract}

Keys words: Female: male ratio, parental age, reproductive performance, quail

\section{Introducción}

La performance reproductiva de la codorniz japonesa (Coturnix coturnix japónica) es importante en la gestión integral de la producción de estas aves, que destacan por su precocidad y por su buena producción de huevos. Narahari et al. (1988) indica que la edad de los reproductores influye en el peso del huevo, calidad del huevo, incubabilidad y calidad de la codorniz BB. Las codornices hembras inician su periodo reproductivo a los 60 días de edad; pues si bien esta es sexualmente madura desde antes, sus huevos tienen un bajo porcentaje de eclosión, debido al menor desarrollo de la yema (Bissoni, 1993). En los machos, la madurez sexual se alcanza a los 42 días de edad (Lucotte, 1990), pero su capacidad fecundante se inicia a los 50 días de edad, su actividad sexual es muy intensa durante 6 meses y luego desciende gradualmente. Wooddard y Abplanalp (1967) encontraron que los machos de 21 semanas de edad son más activos y agresivos, que los de 61 semanas y las hembras jóvenes se aparean más frecuentemente que las viejas. Sefton y Siegel (1973) indican que la actividad copulatoria es máxima entre las 10 y 30 semanas de edad y luego comienza a declinar. Wooddard y Abplanalp (1971) hallaron que la máxima fertilidad fue alcanzada después del impulso inicial de la producción de huevos y después declino gradualmente hasta que las aves alcanzaron las 32 semanas de edad. Babu et al. (1991) evaluaron codornices desde las 7 hasta las 50 semanas de edad, encontrando que los porcentajes de fertilidad e incubabilidad fueron menores al inicio de postura (7 a 10 semanas), luego fueron significativamente altos de 11 a 46 semanas de edad y después comenzaron a declinar de allí en adelante. Othman et al. (2014) evaluaron tres diferentes edades de codornices reproductoras: menores

\footnotetext{
${ }^{1}$ Ingeniero Zootecnista. Práctica Privada.

${ }^{2}$ Departamento Académico de Producción Animal Facultad de Zootecnia-UNALM E-mail: mcumpa@lamolina.edu.pe
} 
de 20 semanas, de 20 a 32 semanas y mayores de 32 semanas de edad, hallando que las aves de edad intermedia tenían una fertilidad e incubabilidad mayores que las aves de otras edades. Genchev (2010) encontraron una mayor fertilidad e incubabilidad en codornices de 2 a 4 meses de edad de producción en comparación con codornices de 1 y 5 meses de edad de producción. En general, diversas investigaciones han determinado que la fertilidad e incubabilidad disminuían significativamente conforme avanza la edad de las codornices reproductores (Seker et al.,2004a; Seker et al.,2004 ; Dudusola, 2013). En cuanto a los ratios de apareamiento hembra: macho, Wooddard y Abplanalp (1967) evaluando relaciones de 1 hasta 6 hembras por macho, encontraron que con relaciones de 1:1 y 2:1 se obtuvieron los mayores niveles de fertilidad. Narahari et al. (1988) no hallaron diferencias tanto en fertilidad como en incubabilidad empleando proporciones de 2:1 hasta 5:1 hembras por macho. Ipek et al. (2004) reportan mayores niveles de fertilidad con las proporciones de 2 y 3 codornices hembras por macho, en comparación con el empleo de 1 y 5 hembras por macho. Asimismo los niveles de fertilidad fueron más altos con reproductores de 11-14 y 15-18 semanas que con aves de 7-10 y de 19-22 semanas de edad. Narine et al. (2013) evaluaron ratios de apareamiento y hallaron mayores niveles de fertilidad empleando 1 y 2 hembras por macho, que cuando emplearon 3, 4 o 5 hembras por macho. Ali et al. (2013) reportan mayores niveles de fertilidad con la proporción de 1:1 (79\%), seguido por los grupos 2:1 (74\%), mientras que los de 3:1 y 4:1 hembras por macho obtuvieron $70 \%$. Igualmente los mayores porcentajes de incubabilidad se obtuvieron con la proporción de 1 hembra por macho. Dere et al. (2009) reportaron que no existen diferencias en porcentajes de incubabilidad entre codornices reproductoras de 3 y 6 semanas de edad.

El objetivo de la presente investigación es determinar los efectos de tres diferentes relaciones hembra: macho y dos edades de codornices reproductoras sobre la producción de huevos, fertilidad e incubabilidad para la producción de codornices BB.

\section{Materiales y métodos}

El presente estudio se llevó a cabo en la Unidad Experimental de Avicultura de la Universidad Nacional Agraria La Molina y tuvo una duración de 4 meses. Se utilizaron 98 codornices reproductoras de 21 semanas de edad (78 hembras y 20 machos) y 116 codornices reproductoras de 47 semanas de edad ( 92 hembras y 24 machos) para la producción de huevos fértiles. Los tratamientos fueron 6 y se formaron de la combinación de tres relaciones de hembras: macho $(\mathrm{H}: \mathrm{M})$ y dos grupos de edades de reproductoras. Los tratamientos evaluados fueron: T1, $3 \mathrm{H}: 1 \mathrm{M}$ (21 semanas); T2, $3 \mathrm{H}: 1 \mathrm{M}$ (47 semanas); T3, $4 \mathrm{H}: 1 \mathrm{M}$ (21 semanas); T4, $4 \mathrm{H}: 1 \mathrm{M}$ (47 semanas); T5, $5 \mathrm{H}: 1 \mathrm{M}$ (21 semanas); T6, $5 \mathrm{H}: 1 \mathrm{M}$ (47 semanas). El alimento y agua fueron suministradas ad libitum a las codornices reproductoras. Para la formulación del alimento de codornices reproductoras se siguieron las recomendaciones del NRC (1994). Las codornices reproductoras de todos los tratamientos fueron alimentadas con una dieta de reproductoras conteniendo $19.78 \%$ de Proteína cruda; 2.95 Mcal de $\mathrm{EM} / \mathrm{Kg}$ de alimento; $2.93 \%$ de calcio y $0.40 \%$ de fosforo disponible, durante todo el experimento.

Se realizaron 7 incubaciones y para cada una se tuvo la siguiente secuencia: Los huevos fueron recolectados una vez al día y se seleccionaron aquellos aptos para incubación. Los huevos se marcaron de acuerdo al tratamiento $\mathrm{y}$ repetición respectiva, y luego se almacenaron hasta por un máximo de 7 días, antes de su carguío en la incubadora. Estos huevos eran colocados en las bandejas, en posición vertical con la punta aguda hacia abajo y fueron cargados en la incubadora. Los primeros 14 días de incubación se les proporciono a los embriones una temperatura de $37.5^{\circ} \mathrm{C}$ y una humedad relativa de 58 a $60 \%$. Se realizaban volteos de los huevos cada dos horas hasta el día 15 de incubación en que eran transferidos a la nacedora. Los embriones en la nacedora recibieron una temperatura de $36.7^{\circ} \mathrm{C}$ y una humedad relativa de $65 \%$. La ventilación durante todo el período de incubación (17 días) era forzada por ventiladores. Se contabilizaron las codornices BB para determinar los porcentajes de incubabilidad. Al final del período de incubación, los huevos no eclosionados fueron abiertos para el control de la fertilidad de los huevos y así determinar: fertilidad e incubabilidad. Los porcentajes de postura, fertilidad e incubabilidad fueron calculados por los siguientes métodos:

Postura $(\%)=$ (número de huevos puestos/ número de codornices reproductoras) $\mathrm{x} 100$

Fertilidad $(\%)=$ (número de huevos fértiles/ número de huevos incubados) $\times 100$

Incubabilidad $(\%)=$ (número de codornices BB nacidas/ número de huevos incubados) x 100

El estudio se realizó bajo el Diseño de Bloques completamente randomizado con arreglo factorial $3 \times 2$ (3 relaciones hembra: macho $\mathrm{x} 2$ edades de reproductores). Los 6 tratamientos se repitieron en cada uno de los 7 bloques (incubaciones). El modelo aditivo lineal empleado es:

$\mathrm{Y}_{\mathrm{ijk}}=\mu+\mathrm{R}_{\mathrm{i}}+\mathrm{E}_{\mathrm{j}}+(\mathrm{R} * \mathrm{E})_{\mathrm{ij}}+\mathrm{B}_{\mathrm{k}}+\mathrm{eijk}$

Dónde:

$\mathrm{Y}_{\mathrm{ijk}}=\mathrm{ijk}$ - ésima observación,$\mu=$ media general, $\mathrm{R}_{\mathrm{i}}=$ efecto de la i-ésima relación hembra: macho, $\mathrm{E}_{\mathrm{j}}=$ efecto de la j-ésima edad, $\left(R^{*} E\right)_{i j}=$ efecto de la interacción hembra: macho por la edad, $\mathrm{B}_{\mathrm{k}}=$ efecto del k- ésimo bloque, $\mathrm{e}_{\mathrm{ijk}}=$ error experimental.

Los datos obtenidos fueron sometidos a análisis de varianza 
usando el programa estadístico Statistical Analysis System (SAS, 1999). Para la comparación de medias se utilizó la Prueba de Duncan (Duncan, 1955). Para la estabilización de la variancia, los valores expresados en porcentajes fueron transformados en valores angulares, empleando la siguiente formula: Arcoseno $\sqrt{ }(\mathrm{Yi} / 100)$ (Steel y Torrie, 1985).

\section{Resultados y discusión}

El porcentaje de postura, fertilidad e incubabilidad de cada tratamiento se muestran en la Tabla 1. Se puede observar que no hubo efecto de la relación hembra: macho, ni de la edad de las codornices reproductoras sobre el porcentaje de postura, pero se encontró un efecto altamente significativo de la interacción relación hembra: macho con la edad.

Tabla 1. Efecto de la relación hembra: macho y la edad de los reproductores sobre los parámetros reproductivos de la Codorniz Japonesa.

\begin{tabular}{|c|c|c|c|c|}
\hline & Tratamientos & Postura (\%) & Fertilidad (\%) & Incubabilidad (\%) \\
\hline \multirow{9}{*}{$\begin{array}{l}\text { Efecto de la Relación } \\
\text { Hembra: Macho }\end{array}$} & 1 & $75.86^{\mathrm{a}}$ & $90.89^{a b}$ & $63.16^{\mathrm{a}}$ \\
\hline & 2 & $64.91^{\mathrm{b}}$ & $93.31^{\mathrm{a}}$ & $66.86^{\mathrm{a}}$ \\
\hline & 3 & $63.78^{b}$ & $92.27^{\mathrm{a}}$ & $66.34^{\mathrm{a}}$ \\
\hline & 4 & $73.39^{a}$ & $91.52^{\mathrm{a}}$ & $64.32^{\mathrm{a}}$ \\
\hline & 5 & $72.57^{\text {a }}$ & $88.18^{\mathrm{b}}$ & $59.91^{\text {a }}$ \\
\hline & 6 & $68.73^{\mathrm{b}}$ & $92.04^{\mathrm{a}}$ & $65.20^{\mathrm{a}}$ \\
\hline & 03:01 & $70.38^{\mathrm{a}}$ & $92.10^{\mathrm{a}}$ & $65.01^{\text {a }}$ \\
\hline & 04:01 & $68.59^{\mathrm{a}}$ & $91.90^{\mathrm{a}}$ & $65.33^{\mathrm{a}}$ \\
\hline & 05:01 & $70.65^{\mathrm{a}}$ & $90.11^{\mathrm{a}}$ & $62.56^{\mathrm{a}}$ \\
\hline Efecto de la Edad de los & 21 & $70.74^{\mathrm{a}}$ & $90.45^{b}$ & $63.14^{\mathrm{a}}$ \\
\hline Reproductores (semanas) & 47 & $69.01^{\mathrm{a}}$ & $92.29^{\mathrm{a}}$ & $65.46^{\mathrm{a}}$ \\
\hline \multicolumn{5}{|c|}{ Probabilidad } \\
\hline Bloques & & $* *$ & $* *$ & $*$ \\
\hline Hembra: Macho & & NS & NS & NS \\
\hline Edad & & NS & $*$ & NS \\
\hline $\mathrm{H}: \mathrm{M} * \mathrm{Edad}$ & & $* *$ & NS & NS \\
\hline
\end{tabular}

T1: 3 H: 1 M (21 semanas); T2: 3 H: 1 M (47 semanas); T3: 4 H: 1 M (21 semanas); T4: 4 H: 1 M (47 semanas); T5: 5 H: 1 M (21 semanas); T6: 5 H: 1 M (47 semanas)

En promedio, las aves en una relación de 5 hembras por macho, mostraron un mayor porcentaje de postura, comparativamente con los obtenidos en las otras proporciones. Asimismo, las aves de menor edad, en promedio, tuvieron un mayor porcentaje de postura que la de mayor edad, esto debido a que las codornices inician la puesta a los 45 días de edad, llegando al pico de postura (90 a $95 \%$ ) a los 70 días de edad y a partir de este pico comienza a descender la curva de postura, razón por la cual las aves de 21 semanas tienen una mayor producción que las de 47 semanas.

En el porcentaje de fertilidad se puede observar diferencias significativas entre las edades evaluadas, mas no se observaron diferencias significativas entre las relaciones de apareamiento, ni en la interacción relación hembra: macho por la edad. En promedio, las aves en una relación de 3 hembras por macho tuvieron un mayor porcentaje de fertilidad que las aves en las proporciones de 4 y 5 hembras por macho, pero no se encontraron diferencias significativas entre estas proporciones evaluadas. Estos resultados concuerdan con lo reportado por Narahari et al. (1988) quienes no hallaron diferencias significativas en las relaciones de 3, 4 y 5 hembras por macho. La fertilidad de los machos no se vio afectada por el aumento del número de hembras para el apareamiento, por lo que se desprende que se mantuvieron adecuadas las características de calidad del semen, motilidad espermática y concentración de semen. Las aves de 47 semanas tuvieron en promedio un porcentaje de fertilidad significativamente mayor que las aves de 21 semanas de edad. Estos resultados difieren de lo obtenido por Wooddard y Abplanalp (1967), Sefton y Siegel (1973) y Dudusola (2013) quienes hallaron una fertilidad significativamente mayor en aves jóvenes comparadas con aves mayores de 32 semanas. De otro lado, Babu et al. (1991) reportaron que la fertilidad de la codorniz se mantiene en un nivel estable desde las 11 hasta las 46 semanas de edad y que a partir de esta edad muestra un descenso. La mayor fertilidad obtenida en los lotes de mayor edad podría deberse a una mayor frecuencia de apareamiento en el caso de los machos y una mayor calidad del huevo, comportamiento y una mayor sobrevivencia de los espermatozoides en las células espermáticas del oviducto en el caso de las hembras reproductoras. La fertilidad promedio de todo el experimento fue de $91.37 \%$, valor que es mayor que los reportados por Alguíar (1998), Flores (1998) y Alejandro (2000), pero menor que lo encontrado por Montalvo (1999).

Para el porcentaje de incubabilidad no se encontraron diferencias significativas entre las relaciones hembras por macho, ni entre edades reproductivas, ni en la interacción relación hembra: macho por la edad. En promedio, las tres relaciones hembras: macho produjeron porcentajes de incubabilidad bastante similares. Estos resultados 
concuerdan con lo hallado por Wooddard y Abplanalp (1967) quienes empleando relaciones desde 1 hasta 6 hembras por macho, hallaron que estas proporciones no tienen efectos sobre las respectivas incubabilidades de los huevos fértiles. No se encontró efecto de las dos edades sobre la incubabilidad, estos resultados concuerdan parcialmente con lo hallado por Wooddard y Abplanalp (1967) quienes estudiando codornices de 9, 10, 25, 43 y 70 semanas de edad y apareándolas en todas las combinaciones posibles de edad, encontraron que la edad de los machos no afecta la incubabilidad, pero si es afectada por la edad de las hembras. Esta diferencia en el caso de las hembras, con los resultados del presente estudio, se puede explicar si tenemos en cuenta que las líneas de codornices, las condiciones medio ambientales y de control genético no necesariamente son las mismas que se emplearon en el presente estudio, pero que sin embargo ejercen efecto sobre la incubabilidad. La edad de los reproductores no afecto la incubabilidad, lo que probablemente se debió a que se mantuvo la calidad de la cáscara del huevo y la calidad interna del huevo que le permitió a los embriones provenientes de madres de mayor edad una buena tasa de sobrevivencia.

En promedio las aves de 21 semanas de edad, produjeron una menor incubabilidad de codornices BB que las aves de 47 semanas de edad. Los tratamientos 2 y 3 tuvieron los mayores porcentajes de incubabilidad relacionado con los mayores porcentajes de fertilidad obtenidos en la presente investigación. En promedio, las aves en proporciones de 3 y 4 hembras por macho exhibieron mayores niveles de incubabilidad en comparación con la obtenida en la relación de 5 hembras por macho, pero no hubo diferencias significativas entre estas proporciones. El promedio general de todo el experimento fue de $64.30 \%$, valor que es similar a lo reportado por Alejandro (2000), pero menor a lo obtenido por Flores (1998) y Montalvo (1999).

\section{Conclusiones}

Bajo las condiciones en las que se ha llevado a cabo el presente estudio, los parámetros reproductivos de producción de huevos, fertilidad e incubabilidad fueron similares en las relaciones hembra: macho evaluadas. Las codornices de 47 semanas de edad tuvieron una fertilidad significativamente mayor que las aves de 21 semanas de edad.

\section{Literatura citada}

Alejandro, M. 2000. Evaluación de normas nutricionales en el comportamiento productivo y reproductivo de la codorniz japonesa (Coturnix coturnix japónica L.). Tesis Ingeniero Zootecnista. UNALM. Lima - Perú. 63 pp.

Alguiar, P. Efecto de la iluminación artificial con rayos amarillos y azules en el rendimiento reproductivo de la codorniz (Coturnix coturnix japónica L.). Tesis Ingeniero Zootecnista. UNALM. Lima - Perú. 71 pp.
Ali, U., Khan, S., Rafiullah, S.K., Chand, N. Shah, Z., Akhtar, A. and Tanweer, J. 2013. Effect of Male to female ratio and vitamin - E Selenium on fertility, hatchability and hatched chick weight of quail breeders. Sarhad Journal of Agriculture. 29(3): 441-447.

Babu, R., Prabakaram, R., Thangavel, K., Mujeer, A. and Sundarasu, V. 1991. Effect of aging on fertility and hatchability in Japanese quails. Journal of Veterinary and Animal Sciences. 22 (1): 12-15.

Bissoni, E. 1993. Crianza de la Codorniz. Ed. Albatros. Buenos Aires. Argentina. 111 p.

Dere, S., Inal, S., Caglayan, T., Garip, M. Y Tilki, M. 2009. The Effects of parent age, egg weigth, storage length and temperature on fertilitiy and hatchability of Japanese quails (Coturnix coturnix japonica) eggs. Journal of Animal and Veterinary Advances 8(7): 12891291.

Dudusola, I.O. 2013. The effect of parental age and weight on fertility, hatchability and day-old chick weight of Japanese quail (Coturnix coturnix japónica). Standard Research Journal of Agricultural Sciences 1(2): 13-16.

Flores, J. 1998. Efecto de diferentes niveles de Zinc Bacitracina en el rendimiento productivo y reproductivo de la codorniz japonesa (Coturnix coturnix japónica L). Tesis Ingeniero Zootecnista. UNALM. Lima - Perú. 78 pp.

Genchev, A. 2010. Effect of age upon the reproductive performance of Japanese quails. Agricultural Science and Technology 2 (1): 9-13.

Ipek, A., Sahan, U. y Yilmaz, B. 2004. The effect of live weight, male to female ratio and breeder age on reproduction performance in Japanese quails (Coturnix coturnix japónica). South African Journal of Animal Science 34 (2): 130-134.

Lucotte, G. 1990. La Codorniz, Cría y Explotación. Ediciones Mundi-Prensa. Madrid. 107 p.

Montalvo, M. 1999. Comportamiento productivo y reproductivo de codornices (Coturnix coturnix japónica) en postura, alimentadas con algarrobo (Prosopis palida). Tesis Ingeniero Zootecnista. UNALM. Lima - Perú. 133 pp.

Narahari, D., Mujeer, A., Thangavel, A., Ramamurthy, N., Viswanathan, S., Mohan, B., Muruganandan, B., Sundararasu, V. 1988. Traits influencing the hatching performance of Japanese quail eggs. British Poultry Science 29 (1): 101 - 112.

Narine, D., Aygun, A. y Sari, T. 2013. Effects of cage type and mating ratio on fertility in Japanese quails (Coturnix coturnix japonica) eggs. Agricultural Science Development 2(1): 4-7.

National Research Council. 1994. Nutrient Requirements of Poultry. Ninth Revised Edition. National Academy Press. Washington D.C. 
Othman, R. A., Amin, M.R., Rahman, S. 2014. Effects of egg size, age of hen and storage period on fertility, hatchability, embryo mortality and chick malformations in eggs of Japanese quail (Coturnix coturnix japonica). Journal of Agriculture and Veterinary Science 7 (1): 101 106.

Sefton, A. and Siegel, P.B. 1973. Mating behavior of Japanese quail. Poultry Science. 52: 1001-1007.

Seker, I., Kul, S. and Bayraktar. 2004a. Effects of parental age and hatching egg weight of Japanese quails on hatchability and chick weight. International Journal of Poultry Science 3(4): 259-265.

Seker, I., Ekmen, F. y Kul, S. 2004b. The effects of parental age and mating ratio on egg weight, hatchability and chick weight in Japanese quail. Journal of Animal and Veterinary Advances 3(7): $424-430$.

Wooddard, A. y Abplanalp, H. 1967. The effects of mating ratio and age on fertility and hatchability in Japanese quail. Poultry Science 46 (2): 383-388 p.

Wooddard, A. y Abplanalp, H. 1971. Longevity and reproduction in Japanese quail under stimulatory lighting. Poultry Science 50 (3): 688-692 p. 